\title{
ADHS bei Erwachsenen: eine Herausforderung für die Gesundheitsversorgung
}

\author{
Die Aufmerksamkeitsdefizit-Hyperaktivitätsstörung (ADHS) bei Erwachsenen ist eine \\ Krankheit, deren Folgen Gesundheitswesen und Volkswirtschaft erheblich belasten. \\ Doch das Leiden findet wenig Beachtung. Dies will die Schweizerische Fachgesell- \\ schaft für ADHS ändern. Auf lokaler Ebene ist die Schaffung interdiziplinärer Netz- \\ werke angesagt.
}

\section{Christophe C. Kaufmann}

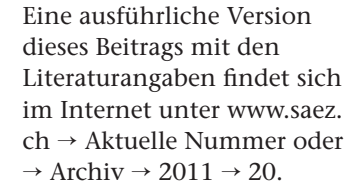
dieses Beitrags mit den Literaturangaben findet sich im Internet unter www.saez. $\mathrm{ch} \rightarrow$ Aktuelle Nummer oder $\rightarrow$ Archiv $\rightarrow 2011 \rightarrow 20$.

Die Aufmerksamkeitsdefizitstörung - mit oder ohne Hyperaktivitätsstörung (ADHS) - ist besonders bei Erwachsenen eine oft verkannte Diagnose. Sie würde eigentlich besser «fortbestehendes Regulierungsdefizit der Aufmerksamkeit, Impulsivität, des Bewegungsdranges und des emotionalen Gleichgewichts und des Sozialverhaltens» heissen.

Es gibt auf Schweizer Verhältnisse zugeschnittene [2]* und internationale [1] diagnostische Empfehlungen sowie Manuale für die klinische Arbeit $[3,4,5$, 6 , 7]. Weltweit sind 3-4\% der Bevölkerung [10] davon betroffen - es gibt also wohl 4-mal mehr ADHSKranke als Borderlinepatienten (1-2\%) [10], 2-mal so viele wie Schizophreniepatienten (2\%) und ähnlich viele wie Patienten mit Depression (4\%). 80\% davon sind anderweitig psychisch krank, und gehören somit zu den schwierigeren psychisch Erkrankten. [17, 26]

\section{Die ADHS-Welle kommt erst noch}

Bis vor kurzem völlig ignoriert, liegt die Zahl der diagnostizierten Fälle, jedenfalls bei Erwachsenen, noch weit hinter der Prävalenz zurück: Die ADHS-Welle kommt also erst noch, da die Diagnose zukünftig mit einem späteren anamnestischen Krankheitsbeginn, auch noch zwischen 7 und 12, vereinbar sein wird. Zudem ziehen sich ADHS-Betroffene gegenseitig an und haben mehr Kinder als nicht Betroffene.

\section{Eine vernachlässigte Risikogruppe}

ADHS-Betroffene sind oft «Heavy users» des Gesundheitssystems. Sie haben eine Vielfalt von psychischen und physischen Beeinträchtigungen und leben auch oft gefährlich und ungesund (s. Kasten auf Seite 762).

Die geforderten Fachgebiete sind: Gynäkologie und Geburtshilfe, Kinder- und Jugendmedizin, Neurologie, Psychiatrie, Allgemein- und Familienmedizin, Dermatologie und Venerologie, Präventiv- und Arbeitsmedizin, Gerichtsmedizin und Begutachtungsmedizin sowie Public Health, ferner Behörden, Justiz, Bildungs- und Rehabilitationswesen. Und natürlich die Versicherer.

In unserem Land müsste eigentlich eine solche Risikogruppe besonders gut erfasst, erforscht und betreut werden. Das Gegenteil trifft zu.

\section{Eine unfassbare Erkrankung?}

ADHS bei Erwachsenen ist ein komplexes Gesamtbild biologischer und psychologischer Zeichen, die sich qualitativ und quantitativ von den Symptomen der Kindheit unterscheiden und sehr weit von dem entfernt sind, was der Volksmund unter «Hyperaktivität» versteht. Die klinische Spezifizität lässt sich kaum von der Art der einzelnen Symptome, wohl aber von deren Verknüpfung, Häufung und Ausprägung ableiten [12]. Das klassische Zustandsbild (mangelnde Aufmerksamkeit, mangelnde Kontrolle über Impulsivität und Bewegungsdrang) ist nicht zwingend.

Bei Hyperfokussierung auf Spannendes (z. B. Videospiele) verschwindet scheinbar das Aufmerksamkeitsdefizit. Die Hyperaktivität ist variabel ausgeprägt und mit dem Alter rückläufig, also auch nicht überall anzutreffen. Die Symptome können auch durch Kompensationsmechanismen überdeckt werden [12] («Ich bin nicht vergesslich, ich muss nur alles aufschreiben»).

Nur 15\% der Betroffenen sind bei Volljährigkeit völlig ausgeheilt, bei den meisten bleibt etwas zurück, dessen Bedeutung von belanglos bis schwer behindernd geht und sich bei zwei Drittel der früher Betroffenen objektivieren lässt [18]. Viele diagnostizierte Erwachsene sind schon seit der Kindheit vorbelastet durch vermehrte traumatogene Situationen («Broken home», vermehrte Demütigungen, Ausgrenzung), späte Schliessmuskelkontrolle, behinderte, verwirrende Ausdrucksformen (unleserliches Schriftbild, miserable Rechtschreibung, undeutliche Aussprache, sprunghafter Gedankenlauf, mangelhafte Intonationsmodulation usw.).

Es handelt sich mehrheitlich um ein multimodales Erbleiden [13]. Die Eltern der erkrankten Kinder sind deshalb oft selber von ADHS betroffen, was bis anhin $\mathrm{zu}$ wenig beachtet wurde. Das Leiden sollte interdisziplinär und transgenerationell angegangen werden.

\section{Behandlung}

Die Behandlung muss individuell angepasst werden. Nicht jeder ADHS-Patient braucht dieselbe Behandlung - nicht jeder braucht sie ständig. Die medikamentöse Behandlung ist zwar oft wesentlich, aber 
nicht ausreichend. $[15,16]$ Man kann auch lediglich in gewissen Lebensphasen auf Psychostimulanzien zurückgreifen, z.B. wenn ein Unfall den Ausgleich durch Sport verunmöglicht, wenn die Symptomatik durch Nikotinentzug stärker zutage tritt, oder wenn

\section{«Die klinische Spezifizität lässt sich kaum von der Art der einzelnen Symptome, wohl aber von deren Verknüpfung, Häufung und Ausprägung ableiten.»}

man sich beruflich in einer kritischen Umbruchphase befindet, in der die Kompensationsmechanismen nicht mehr ausreichen.

Dass Methylphenidat (MPH) als Betäubungsmittel gilt, erschwert natürlich dessen Verordnung. Zudem schreiben Novartis und Swissmedic Beschränkungen vor, die sich eher von wirtschaftlichen und polizeilichen denn von klinischen Überlegungen ab-

\section{ADHS bei Erwachsenen: gravierende Folgen der Unterversorgung}

Bei angemessener Behandlung ist meistens eine beachtliche Verringerung der Auswirkungen der Krankheit zu erwarten. Diese äussern sich wie folgt:

\section{«Schwierige» Patienten}

Schlechte Compliance, verfehlte Termine, Therapieabbrüche, gesetzwidriges Verhalten im Jugend- und jungen Erwachsenenalter [18]; früh auftretendes und schwer zu behandelndes Suchtverhalten $[9,18,20,26]$ (Alkohol, Haschisch, Spiel, Computer usw.); «unverbesserliches» Risikoverhalten (riskantes Geschlechtsleben, Extremsport, Raserei auf Strasse und Pisten, Spiel, Diebstahl, Gewalttätigkeit) [18, 19, 20, 26]; vierfach erhöhtes Risiko für sexuell übertragbare Krankheiten [26]; verfrühte, ungewollte Schwangerschaft bei zwei Dritteln der betroffenen Frauen [9, 20, 21], entsprechend mehr Abtreibungen (diese Toten werden nicht gezählt).

\section{Benachteiligte Patienten}

Traumatisierungsgefahr schon zu Hause, aber nicht vermehrt PTBS, dafür Dissoziations- und Angststörungen, Ablehnungsgefahr durch Gleichaltrige [26]; verspätete Hirnreifungsprozesse: mehr Spätentwickler [22]; vermehrte Ausdrucksschwierigkeiten (unlesbare Schrift, schlechte Rechtschreibung undeutliche Aussprache, sprunghafter Gedankengang, schwallartiges Reden); häufigere Invalidität; Verminderung der Leistungsberechtigung «wegen Kooperationsmangel».

\section{«Schwere» Patienten}

Häufigere Krankenhausaufenthalte und seelische Krisen [28]; viel häufiger Beschwerden am Bewegungsapparat, an den oberen Atemwegen, am Magen-Darm-Trakt sowie Stoffwechselstörungen [24, 26]; kardiovaskuläre Leiden, Obesitas permagna und Schlafapnoesyndrom, schwere chronische Schlafstörungen (DD: restless legs!) sind die Regel.

\section{Gescheiterte Patienten}

Häufigere Rückkehr ins Elternhaus [28] (verfehlte Emanzipation); alleinerziehende Eltern; fast verdoppelte Scheidungsrate [24]; mehr Partnerschaftskrisen [28]; mehr akademische und berufliche Misserfolge (Disziplin- und Lernschwierigkeiten) [20]; Stellenverluste und Arbeitslosigkeit [18,27]; finanzielle Probleme [18]; Inanspruchnahme sozialamtlicher Hilfe; vormundschaftliche Massnahmen; Lebensmissmut [18]; ca. achtfach erhöhte Rate von Selbstmordversuchen nach dem 18. Lebensjahr [9]. leiten lassen. Bei Einnahme therapeutischer Mengen per os kommt es aber auch mit MPH nicht zur Abhängigkeit, es verhütet offenbar sogar deren Auftreten bei exponierten Jugendlichen. Die Aufnahme in die Spezialitätenliste durch das BAG ist nun ein wichtiger Schritt, der einer jahrelangen irrationalen, altersdiskriminierenden und somit $\mathrm{m}$. E. verfassungswidrigen Rationalisierung [17] wenigstens in etwa einem Drittel der Fälle ein Ende setzen kann. In den meisten Fällen bleibt die Behandlung off-label, wird also nicht ohne weiteres von den Kassen finanziert und macht die Behandlung für Minderbemittelte allzu oft unzugänglich.

\section{Zur Diagnose}

ADHS wurde vor mehr als hundert Jahren beschrieben, damals jedoch in ihrer Tragweite nicht erkannt [6]. Ausser bei Verwandten von Betroffenen lässt sich die Diagnose bei Erwachsenen nur selten stellen, ohne dass der Betroffene oder der Diagnostiker spezifisch danach suchen. Die Diagnose ist klinisch zu stellen [2]. Die quantifizierten neuropsychologischen Verfahren liefern zwar therapeutisch wertvolle Hinweise, sind aber in ihrem Ergebnis nicht ausschlaggebend [17]. Es besteht auch die Gefahr des Überund Unterdiagnostizierens, insbesondere dann, wenn dem Verlauf nicht genügend Beachtung geschenkt wird.

Eine isolierte ADHS ist eine Ausnahme. In 80\% der Fälle tritt sie in Verbindung mit (weiteren) psychischen Diagnosen auf $[18,19]$. Die entsprechenden Patienten machen wohl 20\% einer ambulanten psychiatrischen Praxis aus [11]. Die Symptomatik dieser Komorbiditäten überschneidet sich manchmal mehr oder weniger ausgeprägt mit jenen der ADHS. Sowohl bei Depressiven wie bei ADHS-Kranken trifft man Aufmerksamkeits-, Konzentrations- und Gedächtnisstörungen an, ebenso ein vermindertes Selbstwertgefühl, Reizbarkeit, Agitiertheit und eine chronische Schlafstörung.

Für die Diagnose braucht es erfahrene Spezialisten, die über gute differentialdiagnostische Fähigkeiten sowie Kenntnis der Komorbiditäten verfügen sollten. Die Kassen fordern, selbst die Weiterbehandlung

\section{Informative Webseiten}

www.adhd-congress.org

(3. Internationaler Kongress über ADHS, Berlin, 28.-29. Mai 2011

www.elpos.ch www.sfg-adhs.ch www.adhs-deutschland.de www.nice.org.uk/page.aspx $? 0=207034$ www.attentiondeficit-info.com www.tdah.be www.tdah-france.fr www.tdahquebec2012.ca

( $2^{\mathrm{e}}$ Congrès International de Langue Française sur le TDAH, 10, 11, 12 juin 2012 Quebec) 
müsse durch solche Spezialisten erfolgen. Damit vermindern sie das ohnehin schmale therapeutische Angebot.

\section{Zu schliessende Lücken}

ADHS, die Aufmerksamkeitsdefizit- und Hyperaktivitätsstörung bei Erwachsenen, ist eine schwerwiegende Krankheit. Sie muss bei der Ärzteschaft und den Behörden mehr Beachtung finden. Als wesentliche Kostenträger im Bildungs-, Sozial-, Justiz- und
Gesundheitsbereich, wo deshalb zur Zeit unnötige Mehrkosten entstehen, sind Kantone und Steuerzahler die grossen Verlierer. Um die Versorgungslücke zu schliessen, sind die Vorstösse Einzelner ungenügend. Es sind fachliche und kantonale Impulse notwendig. (s. Kasten unten). ADHS soll gebührend berücksichtigt werden in Versorgung, Ausbildung und Planung, aber auch statistisch und gesundheitspolitisch sowie im Forschungsbereich.

\section{Vorschläge zur besseren Beachtung von ADHS bei Erwachsenen \\ Ärzteschaft}

Psychiater aller Alterstufen sind bestens informiert, behandeln integriert sowohl auf medikamentöser als auch auf psychotherapeutischer und psychoedukativer Ebene und arbeiten Hand in Hand zusammen mit allen Betreuenden, insbesondere aus dem pädagogischen und sozialberuflichen Bereich.

Hausärzte erfassen ADHS-bedingte Probleme in der Familie, behandeln sie im Rahmen ihrer Kompetenz, geben Anlaufstellen an. Bei der Abklärung zur Fahrtauglichkeit berücksichtigen sie das Vorliegen von ADHS und verzögern wo nötig die Zulassung zur Fahrprüfung.

Pädiater weisen auch Eltern von diagnostizierten oder ADHS-verdächtigen Kindern an eine Abklärungsstelle, erfassen auch ADHS-verdächtige Jugendliche ohne Schulschwierigkeiten und Hyperaktivität, und benutzen die entsprechenden Screening-Fragebogen.

Gynäkologen und Venerologen weisen bei Appellsymptomen die Patientinnen zu einer Abklärungsstelle für ADHS, besonders in den Risikogruppen Frühschwangerschaft, Schwangerschaftsabbruch und Geschlechtskrankheiten (HIV inbegriffen).

Lungenärzte denken bei starken Rauchern und bei obstruktivem Apnoesyndrom der oberen Atemwege an die Möglichkeit eines zugrundeliegenden ADHS (aber auch an Psychotrauma).

Internisten und Diabetologen denken bei Fettleibigkeit auch an ADHS.

Drogenspezialisten denken an die Möglichkeit von ADHS, klären die Diagnose ab und erwägen die Behandlungsindikation.

Chirurgen denken bei wiederholten Unfällen an ADHS und verweisen die Patienten zur Abklärung.

Die Regionalärztlichen Dienste der IV sind bestens über ADHS informiert und berücksichtigen die spezifischen Schwierigkeiten von ADHSBetroffenen bei der Beurteilung des Invaliditätsgrades, der Rehabilitationsmöglichkeiten und der Rehabilitationsmodalitäten.

\section{Behörden}

Kanton

Auf- und Abklärungsstellen für ADHS aller Altersstufen mit kompetentem Personal. (Sinnvollerweise sind diese Instanzen auch für die Abklärung von Traumata kompetent und arbeiten mit einem weiten Netz von komplementären Instanzen zusammen.)

Zwei Jahre besondere Betreuung für alleinerziehende Mütter und ihr Kind in den Monaten vor und nach der Geburt (vermindert gewaltsames Verhalten bei den Enkeln).

Finanziert, bildet aus und stellt ADHS-spezifisch ausgebildete Begleitpersonen für die Vormundschaftsbehörden an.

Sorgt für einen angemessenen Informationsstand bei Friedensrichtern, Jugend- und Strafrichtern.

Die Bildungsorgane aller Stufen achten auf einen angemessenen Wissensstand beim Lehrpersonal und bei den Schulpsychologen.

Die Verkehrsämter machen Raser und angetrunkene Fahrer auf die Möglichkeit bzw. auf die Notwendigkeit einer Abklärung und evtl. Behandlung des Leidens aufmerksam.

Das Strassenverkehrsgesetz wird angepasst und fördert die Früherfassung und die Verzögerung der Führerscheinabgabe, die Beurteilung der Fahrtüchtigkeit von ADHS-Patienten und fordert die Behandlung, solange die Fahrtüchtigkeit ohne Behandlung nicht gegeben ist. Die Unfallversicherer informieren Fahrer mit wiederholten Bagatellunfällen über die Möglichkeit dieses Leidens.

Sozialämter informieren bei ADHS-Verdacht über Diagnose und Behandlungsmöglichkeiten, besonders bei alleinerziehenden Eltern.

Berufsberater sind über die spezifischen Schwierigkeiten dieser Patientengruppe informiert und beraten sachgemäss.

Die Opferhilfestellen denken bei Gewalt an ADHS und informieren entsprechend.

Die Präventionskampagnen (z. B. gegen Übergewicht, Gewalt, Spielsucht, Suizidversuche) berücksichtigen die Eigenarten dieser Zielgruppe. Statistische Erfassung des Leidens.

Forschungsförderung auf diesem Gebiet. (Zum Beispiel wäre auch folgende Studie in grösserem Rahmen anzulegen: Bei zehn Fahrern, die wegen Fahrens in angetrunkenem Zustand gebüsst wurden und an ADHS litten, wurde MPH eingeführt; darauf verglich man sie mit einer Kontrollgruppe von zehn ebenfalls betroffenen Fahrern ohne Behandlung: In der ersten Gruppen wurden acht, in der zweiten zwei abstinent ... [29].) 
592 d Online

\section{ADHS bei Erwachsenen: eine Herausforderung für die Gesundheitsversorgung}

C.C. Kaufmann

Dr. med, Facharzt für Psychiatrie und Psychotherapie FMH

Korrespondenz: Bd. Pérolles 57, 1700 Fribourg, christophe.kaufmann@hin.ch

Das Aufmerksamkeitsdefizit (mit oder ohne) Hyperaktivität (ADHS) bei Erwachsenen ist eine Krankheit, deren Folgen Gesundheitswesen und Volkswirtschaft seit Jahren erheblich belasten. Nun wird zwar die Behandlung von ADHS auch ab 18 wenigstens weitgehend bezahlt, das Leiden findet aber in Ausbildung, Forschung, Versorgung und Planung immer noch zu wenig Beachtung.

Die Schweizerische Fachgesellschaft für ADHS engagiert sich deshalb für die bessere Beachtung besonders auch der erwachsenen Form von ADHS. Auf lokaler Ebene ist die Schaffung interdiziplinärer Netzwerke angesagt.

Die Aufmerksamkeitsdefizit/Hyperaktivitätsstörung (ADHS) bei Erwachsenen müsste eigentlich auch hierzulande wohlbekannt sein, auch wenn es sich um eine Fehlbezeichnung handelt und besser „Kontrolldefizit der Aufmerksamkeit, Impulsivität, des Bewegungsdranges und der Gefühlslage“ heißen würde.

Es gibt sowohl internationale [1] wie auch auf schweizer Verhältnisse zugeschnittene [2] diagnostische Empfehlungen, sowie Manuale für die klinische Arbeit [3,4,5,6,7

Weltweit sind 3-4\% [10] , davon betroffen - es gibt also wohl viermal mehr ADHS-Kranke als Borderlinepatienten (1-2\%) [10], doppelt so viele wie Schizophreniepatienten (2\%) und ähnlich viele wie Patienten mit Depression (4\%).

$80 \%$ davon sind anderweitig psychisch krank, und bilden somit einen bedeutenden Anteil er schwierigeren psychisch Erkrankten. [17,26]

\section{Nicht selten und immer häufiger?}

Mindestens jeder 30. Bewohner der Schweiz wird durch diese Krankheit z. B. mit Ungehaltenheit, Unstetigkeit, Unkonzentriertheit, Risikoverhalten oder Süchten, Unordentlichkeit, Trägheit, und wiederholtem Misserfolg in den meisten Lebensbereichen belastet. Die Folgen sind z.B. Abtreibung, Geschlechtskrankheiten, Unfälle Arbeitslosigkeit, Ausgrenzung, chronische Leiden, verminderte Rehabilitationsfähigkeit sowie eine achtfach höhere Rate von Selbstmordversuchen bei Erwachsenen[7].

Die bessere Kenntnis des Wesens von ADHS und seines Verlaufs führen dazu, nun auch Fälle (v.a. mit Aufmerksamkeitsdefizit) zu berücksichtigen, in denen die Störung später, zwischen 7 und 12 bemerkt wurde, was tendenziell zu einer Erweiterung der Diagnose und somit der Prävalenz führt, die jetzt auf $7 \%$ geschätzt wird [11]. 
Die wachsende Prävalenz wird auch zudem dadurch gefördert, dass ADHS-Betroffene sich gegenseitig anziehen und mehr Kinder haben, als nicht Betroffene.

\section{Eine vernachlässigte Risikogruppe}

Bei ADHS-Betroffenen, die den DSM-IV Kriterien konform diagnostiziert wurden, bestehen teilweise starke psychische und physische Beeinträchtigungen; sie leben auch oft ungesund und gefährlich (siehe Infobox I Folgen). Sie gehören des öftern zu den „heavy users“ des Gesundheitssystems.

Man hätte annehmen können dass eine solche Risikogruppe besonders gut erfasst, erforscht und betreut würde. Die Bedeutung der medikamentösen Behandlung als wesentlich wenn auch unzureichend ist schon seit Jahren eindeutig $[4,5]$.

Trotzdem hat es viele Jahre gebraucht, bis medikamentöse Komponente der Behandlung nun endlich mindestens teilweise auch nach dem Alter von 18 Jahren von den Grundversicherungen übernommen wird.

Nicht nur finanziell sondern auch menschlich ist diese Tatsache folgenschwer, denn es handelt sich um Menschen, die mit ihrer Kreativität, ihrem Gerechtigkeitssinn, ihrer Begeisterungsfähigkeit und ihrem Humor wertvolle Beiträge in der Gesellschaft leisten könnten.

\section{Jenseits von Aufmerksamkeitsdefizit und Hyperaktivität: eine schwer zu erfassende Krankheit ?}

Weder die Bezeichnung des Leidens als Aufmerksamkeitsdefizit, noch jene als Hyperaktivitätsstörung sind völlig zutreffend: das klassische Zustandsbild von ADHS bei Erwachsenen (mangelnde Aufmerksamkeit, und mangelnde Kontrolle über Impulsivität und Bewegungsdrang) ist nicht zwingend: .Bei Hyperfokussierung auf Spannendes verschwindet scheinbar das Aufmerksamkeitsdefizit, und die Hyperaktivität ist variabel ausgeprägt und mit dem Alter rückläufig, also auch nicht überall anzutreffen, ferner können die Symptome durch Kompensationsmechanismen überdeckt sein [12] .

ADHS bei Erwachsenen erweist sich also als komplexes Gesamtbild biologischer und psychologischer Zeichen, die sich qualitativ und quantitativ von den Symptomen der Kindheit unterscheiden und sehr weit von dem entfernt ist, was der Volksmund unter „Hyperaktivität“ versteht. Es ist ein Leiden, dessen klinische Spezifizität sich kaum von der Art der einzelnen Symptome, wohl aber von deren Häufung und Ausprägung ableiten lässt. [12]

Eine typische aktuelle Klinik reicht aber nicht aus, um die Diagnose zu stellen. Das Zurückreichen der Symptomatik bis in die Kindheit, das Vorkommen in verschiedenen Lebensbereichen, eine bedeutende funktionelle Einschränkung und der Ausschluss anderer Krankheiten, welche die Klinik besser erklären könnten, sind ebenfalls für die Diagnose erforderlich.

Die diagnostischen Kriterien haben sich ebenso wie die Bezeichnung des Leidens (POS, minimal brain dammage, Hyperkinetische Störung, ADHS) im Laufe der Jahre weiterentwickelt und weder diejenigen der hyperkinetischen Störung der ICD-10 noch jene des DSM IV werden wohl die bevorstehende Revision unverändert überleben. 
Es ist eine schillernde Erkrankung: deren Ausprägung und Ausdrucksform stark von psychosozialen Parametern geprägt sind.

$15 \%$ der betroffenen Jugendlichen sind bis zur Volljährigkeit völlig ausheilt, bei den übrigen Erwachsenen sind fliessende Übergänge zum Normalen festzustellen, Erwachsenen, deren leichte, oft residuale Form, keine Diagnose mehr rechtfertigen, weil ein minimaler Schweregrad nicht erreicht wird. Das geht soweit, dass gewisse Autoren darin lediglich den Jägertypus des Menschen sehen, der sich vom sesshaften Bauertypus unterscheidet und also eine Variante der Norm darstellt, die durch Veränderung der gesellschaftichen Norm und Toleranz stigmatisiert wurde. [12] Auch wenn es sich um eine interessante Perspektive handelt, entspricht sie im Wesentlichen nicht dem, was wir in der Sprechstunde antreffen, wo in Anbetracht des Lebenslaufes und der Familienanamnese eine Unterscheidung vom Normalen durchaus eindeutig ist.

\section{Eine lebenslängliche transgenerationelle Belastung}

Die meisten Erwachsenen, die diagnostiziert wurden, sind schon seit der Kindheit auf verschiedenste Weise vorbelastet durch vermehrte traumatogene Situationen („Broken home“, vermehrte Demütigungen, Ausgrenzung), späte Schliessmuskelkontrolle, behinderte Ausdrucksformen (Schriftbild, Rechtschreibung, Aussprache, Gedankenstruktur) etc..

Nur etwa $25 \%$ sind perinatal oder umweltbedingt erworbene Formen [13]. In der Mehrzahl ist ADHS ist ein multimodales Erbleiden. [13]

Daher sind oft auch die Eltern der erkrankten Kinder von ADHS betroffen.

Diesem Tatbestand wurde bis anhin nicht die gebührende Beachtung geschenkt.

\section{Herausforderung für Patienten und Gesellschaft}

ADHS ist, besonders bei Erwachsenen, eine komplexe Diagnose. Sie wird noch viel zu selten korrekt gestellt und bleibt deshalb in etwa neun von 10 Fällen verkannt und medikamentös unbehandelt, wenn man davon ausgeht, dass z.Z.nur 0,3 \% der Bevölkerung Psychostimulanzien erhalten [30], wo doch mindestens 3\% davon profitieren könnten.(Die Anzahl ohne psychostimulante Medikation behandelte ADHS-Erwachsene ist unbekannt, aber wohl noch geringer.)

Bei verfehlter Diagnose gelten die Patienten oft als faul, unstet und rücksichtslos. Meistens zeigen sie sich darüber bestürzt, sind aber ohne Behandlung unfähig, sich zu ändern.

Nicht jeder ADHS-Patient braucht dieselbe Behandlung - nicht jeder braucht sie ständig. Die medikamentöse Behandlung ist zwar oft wesentlich, aber nicht ausreichend. [14,15]

Man kann auch lediglich in gewissen Lebensphasen auf Psychostimulanzien zurückgreifen, z.B. wenn ein Unfall den Ausgleich durch Sport verunmöglicht, wenn die Symptomatik durch Nikotinentzug stärker zu Tage tritt, oder wenn man sich beruflich in einer kritischen Umbruchphase befindet, wo die Kompensationsmechanismen nicht mehr ausreichen.

Weil diese Kompensationsmechanismen oft von Routinen und Drittpersonen und Umfeldbedingungen abhängig sind, sind angepasste ADHS-Patienten oft auffallend veränderungsscheu, trotz ihrer Intoleranz für Langeweile und ihrer Abwechslungsfreudigkeit. 
Mit dem Leiden haben die verschiedensten Fachgebiete der Medizin zu tun (Präventiv- und Arbeitsmedizin, Gynäkologie und Geburtshilfe, Kinder-und Jugendmedizin, Dermatologie und Venerologie, Neurologie, Psychiatrie, Allgemein- und Familienmedizin, Gerichtsmedizin und Begutachtungsmedizin sowie Public Health) .Die Behörden, die Justiz, sowie das Bildungs- und Rehabilitationswesen sind ebenfalls gefordert. Von den Versicherern gar nicht zu sprechen.

Das Leiden sollte also transdisziplinär und transgenerationell angegangen werden

Das Gebiet könnte sich eignen, zum Überdenken der Managed-Care-formen für die Medizin der Zukunft.

\section{Stolpersteine für eine adäquate medikamentöse Behandlung}

Dass Methylphenidat (MPH) als Betäubungsmittel gilt, erschwert natürlich dessen Verordnung. Es ist jedoch wegen des Schwarzmarktes gerechtfertigt, wo die Substanz sehr gewinnreich verkauft bzw. gemischt oder in methamphetamin umgewandelt wird und auch, durch das Suchtpotenzial, das sich ergibt, wenn die Substanz parenteral oder paranasal zu sich genommen wird.

Atomoxetin hat nicht die gleiche Wirkungsbreite, ist also gerade bei Aufmerksamkeitsdefizit keine Alternative [3].

Bei Patienten ohne Suchtanamnese bzw. bei Einnahme verschriebener Mengen per os kommt es aber auch mit MPH nicht zur Abhängigkeit, es verhütete sogar deren Auftreten bei exponierten Jugendlichen.

In der Schweiz ist nur die Pharmaindustrie befugt, Anwendungsbereiche und Dosierungsvorschläge für eine Substanz bei Swissmedic zu beantragen, also weder die Ärzteschaft, noch die Behörden, noch die öffentliche Hand, selbst wenn es im öffentlichen Interesse wäre.

Deshalb hat es solange gedauert: Novartis hat die Indikation ADHS bei Erwachsenen für ihr vierzigjähriges Medikament Ritalin bei Swissmedic gar nie beantragt

Swissmedic hat vor etwas mehr als einem Jahr zwei Formen von MPH (ein Enantiomer (Dexmethylphenidat) von Novartis (Focaline $X R{ }^{\circledR}$ ) und ein weiteres Retardpräparat (Concerta ${ }^{\circledR}$ von Janssen) zur Behandlung des Leidens bei Erwachsenen zugelassen.

Die Aufnahme in die Spezialitätenliste durch das BAG ist ein wichtiger Schritt, der einer jahrelangen irrationalen, altersdiskriminierenden und somit $m$.E. verfassungswidrigen Rationalisierung [16] in einem Teil der Fälle (etwa 30 \%) ein Ende setzt. (Concerta ist noch nicht so weit)

Die zugelassenen Präparate decken leider von der Dosierung und Wirkungsdauer her die Bedürfnisse der Patienten meistens nur teilweise ab.*

In dem Novartis und Swissmedic davon ausgehen, dass maximale Einzeldosis und Tagesdosis sich entsprechen, obschon die notwendige Dosierung individuell angepasst werden muss und stark variiert und obschon die individuell verschiedene Wirkungsdauer einer Einzeldosis nie die 16 Stunden der Wachzeit eines Erwachsenen abdecken, werden 
dem behandelnden Arzt Begrenzungen aufgezwungen, die sich wohl besser politisch, wirtschaftlich und polizeilich denn klinisch begründen lassen.

In den meisten Fällen bleibt die Behandlung off-label, wird also nicht ohne weiteres von den Kassen finanziert und macht die Behandlung für Minderbemittelte oft unzugänglich.

Kassen verweigern die Finanzierung z.B. wenn der individuelle Metabolismus, die erforderliche Dauer der Wirkung oder das Ansprechen auf MPH nicht in das von Swissmedic anerkannte Behandlungsschema passen oder wenn der behandelnde Arzt der Meinung ist, eine (weitere) ausschliesslich medikamentöse Behandlung sei ausreichend. Bei vorliegenden Traumata wird im Compendium 2011 gar vor der Verordung von Focalin XR eine vorgängige Verwahrung in einer Erziehungsanstalt beantragt!

Ferner reagieren nicht alle Patienten auf MPH und/oder Atomoxetin (bei Erwachsenen nicht zugelassen), weshalb weiterhin auch die off-label Verordnung von Dexamphetamin gerechtfertigt sein kann. [3]

\section{Eine Hintergrund- und Verlaufsdiagnose, die übersehen werden kann}

ADHS gilt als Modekrankheit, obschon sie vor mehr als hundert Jahren beschrieben, damals jedoch in ihrer Tragweite nicht erkannt wurde [6]

Die Patienten kommen meist aus anderen Gründen und kennen ihr Leiden nur selten, es sei denn, es handle sich um Verwandte von Betroffenen.:Im Gegensatz zu den auffälligen Kindern wird sich die Diagnose ADHS, d.h. Aufmerksamkeitsdefizit (mit oder ohne) Hyperaktivitätssyndrom bei Erwachsenen nur selten stellen lassen, wenn man nicht spezifisch danach sucht.

Die Diagnose ist klinisch zu stellen [2], weil die quantifizierten neuropsychologischen Verfahren in ihrem Ergebnis nicht ausschlaggebend sind, auch wenn sie therapeutisch wertvolle Hinweise liefern. [17

Weil die Gegenwartssymptomatik an sich unspezifisch ist, besteht auch die Gefahr des Über- und Unterdiagnostizierens, insbesondere dann, wenn dem Verlauf nicht genügend Beachtung geschenkt wird.

Die Diagnose ist weder ex juvantibus zu stellen (es gibt auf Grund des Behandlungsresultats zu viele falsch negative und einige falsch positive Ergebnisse), noch ist ADHS eine Ausschlussdiagnose: sie ist im Gegenteil mit einer «Entweder-Oder-Logik» fast unvereinbar. ADHS tritt eben nur ausnahmsweise isoliert auf, sondern in $80 \%$ der Fälle in Verbindung mit (weiteren) psychischen Diagnosen [18, 19].

\footnotetext{
*Manche wenige brauchen, nach eigenen Erfahrungen, schwächer dosierte und kürzer wirkende Formen ( z. B. um den appetitzügelnden Effekt zur Essenszeit mittags nicht aufrecht zu erhalten), andere, zahlreichere, dagegen brauchen die mehrmalige Einnahme von Retard-Formen, damit eine befriedigende Wirkung über eine ausreichend lange Zeit erreicht wird und überschreiten damit die kostenpflichtige Dosis. Es gibt keine Standarddosierung. In der Regel liegen die MPH Tagesdosen bei Erwachsenen eindeutig unter 1mg/kg.bzw 9 mg/ Std. [19] die Tagesdosis liegt bei meinen Patienten zwischen 2x 2,5 und $120 \mathrm{mg}$

Die von Swissmedic zugelassenen maximale Tagesdosis von 72 mg/d Concerta bzw. 20 mg Focalin XR (in einmaliger Einnahme ) scheint eher markt- und politikorientiert denn klinisch begründet [2]. insbesondere, wenn man das Leiden nicht nur tagsüber, sondern auch abends (wegen Paar- und Erziehungsproblemen sowie Sozialkontakten) behandeln will und sich somit die abzudeckende Zeitspanne auf 10 bis 14 Std beläuft, was mindestens zwei Einnahmen pro Tag erfordert [19].
} 
ADHS Patienten reagieren dabei nicht selten atypisch auf die Psychostimulanzien, indem sie besser den Schlaf finden und eine bessere Schlafqualität mit verbesserten Erwachungsbedingungen feststellen können. [18], wenn das Wirkungsende nicht in die Einschlafzeit fällt und Reboundphänomene bewirkt.

Die Symptomatik dieser Komorbiditäten überschneiden sich manchmal mehr oder weniger weitgehend mit jenen von ADHS, wie bei Depression, bipolarer Störung und emotional instabile Persönlichkeitsstörung vom Borderline Typus oder auch allgemeiner vei persönlichkeitsveränderungen nach psychischen Traumata.

Somit trifft z.B. man sowohl bei Depressiven wie bei ADHS-Kranken (ohne Depression) Aufmerksamkeits- Konzentrations- und Gedächtnisstörungen (ADHS nur wenn's nicht sehr spannend ist) an, ebenso ein vermindertes Selbstwertgefühl, eine Reizbarkeit, Agitiertheit sowie eine chronische Schlafstörung (bei „reinem“ ADHS ohne frühes Erwachen).

So machen die entsprechenden Patienten wohl $20 \%$ einer ambulanten psychiatrischen Praxis aus. [11] Um sie zu diagnostizieren braucht es gute differential-diagnostische Fähigkeiten sowie Kenntnis der Komorbiditäten. Für den Therapeuten ergibt sich dadurch die Notwendigkeit, sich in ein pluridisziplinäres Netzwerk von Therapeuten einzufügen.

ADHS ist unter Erwachsenenpsychiatern erst seit Kurzem ein Thema und wurde lange ignoriert und vernachlässigt. Ganz besonders bei ADHS-Patienten ohne

Schulschwierigkeiten. [12] Nach eigener Erfahrung wurde die Diagnose bei Gutachten auch schon verschwiegen oder ausgeklammert und bei Spitalaufenthalten die Behandlung diskussionslos abgebrochen [32, 33].

\section{Zu schliessende Lücken}

Es gibt keine Statistik, wie viele Tote täglich oder jährlich darauf zurückzuführen sind, dass ADHS bei Erwachsenen so stark unterdiagnostiziert und unterbehandelt wird. Dafür wäre das BAG zuständig. Es scheint aber so, als werde die gegenwärtige Planung der psychiatrischen Versorgung immer noch vorgenommen, ohne dieses Leiden zu berücksichtigen.

Gerade die Kantone sind grosse Verlierer in dieser Geschichte, denn sie sind wesentliche Kostenträger im Bildungs- Sozial- Justiz- und Gesundheitsbereich, wo deshalb zurzeit unnötige Mehrkosten entstehen.

Um die Versorgungslücke zu schliessen, sind die Vorstösse Einzelner ungenügend: es wären fachliche und kantonale Impulse notwendig. (vgl Infobox II: Zielvorschäge)

\section{Zusammenfassend}

ADHS, die Aufmerksamkeitsdefizit- und Hyperaktivitätsstörung bei Erwachsenen ist eine schwerwiegende Krankheit, die in Versorgung, Ausbildung, und Planung, aber auch statistisch und gesundheitspolitisch und im Forschungsbereich bei der Ärzteschaft und den Behörden mehr Beachtung finden muss.

ADHS- Diagnostik und Therapie von Erwachsenen war viel zu lange Brachland. Es zu bearbeiten; ist ein dringliches und lohnendes Ziel, eine Herausforderung sowohl für die betroffenen Patienten wie auch für die Ärzte 
Wollen Sie sich direkt für ADHS bei Erwachsenen engagieren? Willkommen in unserer Fachgesellschaft (www. sfg-adhs.ch) und im Aktionsbündnis zur besseren Beachtung der erwachsenen Form des ADHS (Kontakt über den Autor: christophe.kaufmann@hin.ch)!

\section{Infobox I}

\section{ADHS bei Erwachsenen: Gravierende Folgen der Unterversorgung}

ADHS heilt nur bei $15 \%$ der kindlichen und jugendlichen Patienten vollständig aus, bevor sie erwachsen sind [7], während man früher davon ausging, dies sei die Regel. Bei den übrigen $85 \%$ persistieren in etwa drei von vier Fällen objektive Beeinträchtigungen [18,24,25].

Die Behandlung verhindert nicht alle Folgen von ADHS bei Erwachsenen. Dennoch ist bei einer adäquate Therapie und der besseren Berücksichtigung der Natur des Leidens eine beachtliche Verringerung der gravierenden Auswirkungen der Krankheit zu erwarten. Dies äussert sich z.B. so:

„Schlechte Patienten“(Häufiger schlechte Compliance, verfehlte Termine und vorzeitige

Therapieabbrüche, „Kooperationsmangel“

Gehäuft gesetzwidriges Verhalten im Jugend- und jungen Erwachsenenalter [18];

Tendenz zu, zum Teil früh auftretendem und schwer zu behandelndem Suchtverhalten $[9,18,20,26]$ (Alkohol, Haschisch, Spiel, Computer etc.);

Häufigeres „unverbesserliches“ Risikoverhalten (riskantes Geschlechtsleben, Extremsport, Raserei auf Strasse und Pisten, Spiel, Diebstahl, Gewalttätigkeit) [9, 20,, 21]

Vierfach erhöhtes Risiko für sexuell übertragbare Krankheiten [20];

Verfrühte, ungewollte Schwangerschaft bei zwei Drittel der betroffenen Frauen [9, 20,, 21]

Entsprechend mehr Abtreibungen (diese Toten werden nicht gezählt)

Benachteiligte Patienten Erhöhte psychische Traumatisierungsgefahr schon ab dem Kindesalter. Zwar sind posttraumatische Belastungsstörungen bei ADHS-Patienten nicht häufiger zu beobachten [26], wohl aber andere Traumafolgen (z.B. Dissoziations- und Angststörungen);

Stärkere Ablehnungsgefahr durch Gleichaltrige [26];

Verspätete Hirnreifungsprozesse: mehr Spätentwickler [22];

Vermehrte Ausdrucksschwierigkeiten (unlesbare Schrift, schlechte Rechtschreibung undeutliche Aussprache, sprunghafter Gedankengang, schwallartiges Reden);

Häufigere Invalidität; Häufigere Ablehnungswahrscheinlichkeit wegen Kooperationsmangel

"schwere Patienten: Häufigere Krankenhausaufenthalte und seelische Krisen [28]. mehr als doppelt so häufig wie bei Gesunden kommen bei ADHS] Beschwerden am Bewegungsapparat, an den oberen Atemwegen, am Magen-Darmtrakt sowie Stoffwechselstörungen vor : $[24,26]$ es finden sich häufiger kardiovaskuläre Leiden, Obesitas permagna und Schlafapnoesyndrom, aufgrund schlechter Lebenshygiene;

Schwere chronische Schlafstörungen (DD : restless legs !) sind die Regel.

\section{Gescheiterte Patienten:}

Häufigere Rückkehr ins Elternhaus [28] (verfehlte Emanzipation);

Mehr alleinerziehende Eltern;

Fast verdoppelte Scheidungsrate [24]; mehr Partnerschaftskrisen [28];

Mehr akademische und berufliche Misserfolge mit Disziplin- und Lernschwierigkeiten [20];

häufiger Stellenverluste und Arbeitslosigkeit [18,27

Vermehrt finanzielle Probleme [18];

Höhere Wahrscheinlichkeit, sozialamtliche Hilfe zu beanspruchen;

Häufiger vormundschaftliche Massnahmen;

stärkerer Lebensmissmut [18]; ca. achtfach erhöhte Rate von Selbstmordversuchen ab dem Alter von 18 Jahren [9] 


\section{Infobox II}

\section{Zielvorschläge zur besseren Beachtung von ADHS bei Erwachsenen}

\section{Zuhanden der Aerzteschaft}

Psychiater aller Alterstufen sind bestens informiert, behandeln integriert sowohl auf medikamentöser als auch auf psychotherapeutischer und psychoedukativer Ebene und arbeiten Hand in Hand zusammen mit allen Betreuenden, insbesondere aus dem pädagogischen und sozial-beruflichen Bereich.

Hausärzte erfassen adhs-bedingte Probleme in der Familie, behandeln sie im Rahmen ihrer Kompetenz, geben Anlaufstellen an. Bei der Abklärung zur Fahrtauglichkeit berücksichtigen sie das Vorliegen von ADHS und verzögern wo nötig die Zulassung zur Fahrprüfung.

Pädiater weisen auch Eltern von diagnostizierten oder adhs-verdächtigen Kindern an eine Abklärungsstelle, erfassen auch adhs-verdächtige Jugendliche ohne Schulschwierigkeiten und Hyperaktivität, und benutzen die entsprechenden Screening-Fragebogen. .

Gynäkologen und Venerologen weisen bei Appellsymptomen die Patientinnen zu einer Abklärungsstelle für ADHS, besonders in den Risikogruppen Frühschwangerschaft, Schwangerschaftsabbruch und Geschlechtskrankheiten (HIV inbegriffen).

Lungenärzte denken bei starken Rauchern und bei obstruktivem Apnoesyndrom der oberen Atemwege an die Möglichkeit eines zugrundeliegenden ADHS (aber auch an Psychotrauma)

Internisten und Diabetologen denken bei Fettleibigkeit auch an ADHS.

Drogenspezialisten denken an die Möglichkeit von ADHS, klären die Diagnose ab und erwägen die Behandlungsindikation .

\section{Chirurgen denken bei wiederholten Unfällen an ADHS und verweisen die Patienten zur Abklärung}

Die Regionalärztlichen Dienste der IV sind bestens über ADHS informiert und berücksichtigen die spezifischen Schwierigkeiten von adhs-Betroffenen bei der Beurteilung des Invaliditätsgrades, der Rehabilitationsmöglichkeiten und der Rehabilitationsmodalitäten.

\section{Zuhanden der Behörden}

Kanton :

Auf- und Abklärungsstellen für ADHS aller Altersstufen mit kompetentem Personal. (Sinnvollerweise sind diese Instanzen auch für die Abklärung von Traumata kompetent und arbeiten mit einem weiten Netz von komplementären Instanzen zusammen.)

2 Jahre besondere Betreuung für alleinerziehende Mütter und ihr Kind in den Monaten vor und nach der Geburt . (Vermindert gewaltsames Verhalten bei den Enkeln)

Finanziert, bildet aus und stellt Adhs-spezifisch ausgebildete Begleitpersonen für die Vormundschaftsbehörden an

Sorgt für einen angemessenen Informationsstand bei Friedensrichtern, Jugend- und Strafrichtern. 
Die Bildungsorgane aller Stufen achten auf einen angemessenen Wissensstand beim Lehrpersonal und bei den Schulpsychologen.

Die Verkehrsämter machen Raser und angetrunkene Fahrer auf die Möglichkeit bzw. auf die Notwendigkeit einer Abklärung und evt. Behandlung des Leidens aufmerksam.

Strassenverkehrsgesetz wird angepasst und fördert die Früherfassung und die Verzögerung der Führerscheinabgabe, die Beurteilung der Fahrtüchtigkeit von ADHS-Patienten und fordert die Behandlung solange die Fahrtüchtigkeit ohne Behandlung nicht gegeben ist.

Die Unfallversicherer informieren Fahrer mit wiederholten Bagatellunfällen über die Möglichkeit dieses Leidens

Sozialämtern, informieren bei ADHS-Verdacht über Diagnose und Behandlungsmöglichkeiten, besonders bei alleinerziehenden Eltern

Berufsberater sind über die spezifischen Schwierigkeiten dieser PatientInnen informiert und beraten sachgemäss.

Die Opferhilfestellen denken bei Gewalt an ADHS und informieren entsprechend

Die Präventionskampagnen (z.B.gegen Übergewicht, Gewalt, Spielsucht, Suizidversuche u ä) berücksichtigen die Eigenarten dieser Zielgruppe.

Statistische Erfassung des Leidens.

Forschungsförderung auf diesem Gebiet. **

**Zum Beispiel wäre auch folgende Studie in grösserem Rahmen anzulegen:

Bei zehn Fahrern, welche wegen Fahren in angetrunkenem Zustand gebüsst worden waren und an ADHS litten, wurde MPH eingeführt; darauf verglich man sie mit einer Kontrollgruppe von zehn ebenfalls betroffenen Fahrern ohne Behandlung: In der ersten Gruppen wurden acht, in der zweiten zwei abstinent ... (29)

\section{Wenn Sie mehr über ADHS bei Erwachsenen erfahren möchten, bieten Ihnen folgende Links wertvolle Informationen:}

3. Internationaler Kongress über ADHS, Berlin vom 28 bis 29 Mai 2011

www.adhd-congress.org

www.elpos.ch

www.sfg-adhs.ch (dt.)

www.adhs-deutschland.de (dt)

www.nice.org.uk/page. $a s p x ? o=207034$ (engl.)

www.attentiondeficit-info.com (engl / fr.)

www.tdah.be (fr.)

www.tdah-france.fr (fr)

und der $2^{\text {ème }}$ Congrès International de Langue Française sur le TDAH 10,11,12 juin 2012 im Hôtel Hilton Quebec in Quebec www.tdahquebec2012.ca 


\title{
Bibliographie:
}

1. Stephen V. Faraone et K.V. Antshel: Diagnosing and treating attentions-deficit/hyperactivity disorder in adults, World Psychiatry $2008 ; 7 ; 131-136$ )

2. Baud et al. : Empfehlungen zur Diagnostik und Therapie der AufmerksamkeitsdefizitHyperaktivitätsstörung (ADHS) im Erwachsenenalter, Schweizer Archiv für Neurologie und Psychiatrie, (158) 5, 2007, p 217-224

3. Krause und Krause : ADHS im Erwachsenenalter, Schattauer, 3. Auflage 2009.

4. Russel A. Barkley: Attention-Deficit Hyperactivity Disorder, A Handbook for Diagnosis and Treatment 3. Aufl. Guildford 2006

5. Brian B. Doyle Understanding and Treating Adults with Attention Deficit Hyperactivity Disorder American Psychiatric Publishing Inc. Washington DC 2006, ISBN 1-5862-221-4

6. Bange F, M.C. Mouren-Siméoni: Comprendre et Soigner l'hyperactivité adulte, Dunod, 2è ed. 2009

7. Attention-Deficit/Hy... Disorder in Children and Adults (Advances in Psychotherapy; EvidenceBased Practice) by Annette U. Rickel and Ronald T. Brown ,Hogrefe \& Huber Publishers, Canada, 2007

8. Vincent Annick, Mon Cerveau a encore Besoin de Lunettes - Vivre avec l'Hyperactivité. Les Editions Quebecor, 2010

9. Barkley R. A. Murphy K.R. \& Fisher M: ADHD in Adults, What the Science Says, Guildford, N.Y. 2008

10. Ces chiffres se rapportent aux USA, et proviennent en particulier de Kessler R. et al 2006, Am J of Psychiatry, 163,716-723, Kessler, JOEM 2005 et Faraone JAtt Dis 2005, APA, 2004 ; Oldham, 2005

11. Prof. D. Eich, Zürich, persönliche Mitteillung; für die USA: siehe Barkley R.A. One-DayWorkshop, sfg-adhs-Tagung vom 8.9.08 in Bern.

12. C. Gertsch Le TDAH chez l'adulte, source de handicaps ou identité positive ?, Psychoscope, $11 / 2009$ (30), p 21-25

13. Nigg J.T. What causes ADHD ? New York, Guilford Publications 2006 Dazu noch folgener Hinweis von Medscape:

\begin{abstract}
and not a behavioral disorder.
Deborah Flapan, Director, Medscape Medical News, Chicago.

Deborah Flapan has disclosed no relevant financial relationships.

Jane Hwang, Senior Editor/Photo, Medscape, New York City Jane Hwang has disclosed no relevant financial relationships.
\end{abstract}

New research provides the first direct evidence that attention-deficit/hyperactivity disorder (ADHD) is genetic. In a study published online September 30 in The Lancet, investigators from the University of Cardiff, United Kingdom, say their findings suggest ADHD should be classified as a neurodevelopmental

\section{For more information, see the following resources:}

The Year in Medicine 2010

Ongoing Medscape Medical News Coverage

14. Elsässer, M., Nyberger, E., Stieglitz, R. D. Z. Kognitiv-behaviorale Strategien in der Behandlung der ADHS im Erwachsenenalter Z Psychiat Psychol Psychother vol 58, Nr 1 2010, p 35-44

15. Safren, SA, Perlmann, C.A. Sprich, S., \%Otto, M.W. (2005) Mastering your adult ADHD, Therapist Guide ( zu Deutsch : 2009 Kognitive Verhaltenstherapie der ADHS des Erwachsenenalters (deutsche Bearbeitung von E. Sobanski, M. Schunacher-Stien\& B. Alm) Medizinisch Wissenschaftliche Verlagsgesellschaft

16. Académie des Sciences Médicales, Bulletin des Médecins Suisses, Nr 35 vom 29.8.07 ,S. 1431 (éd. française)

17. Grützemacher H Dt Ärzteblatt 2001: Fallkontrollstudie Kinder unter 15 Jahren.

18. Barkley R. A. , Fisher et al , Milwaukee-Study

19. Schlander M. Schwarz O. Trott GE, Vapiano M, Bonauer N (2006)

20. Barkley R.A. One-Day-Workshop, sfg-adhs-Tagung vom 8.9.08 in Bern. 
21. Biedermann $\mathrm{J}$ et al Functional impairments in adult with self-reports of diagnosed ADHD: $A$ controlled study of 1001 adults in community J. Clin Psychiatry 2006;67:524-40

22. Shaw. P. Et al (2007) ADHD is characterized by a delay in cortical maturation. Proceedings of The National Academy of Sciences, 104,19649-54

23. Biedermann et al 2006, zitiert durch Trott (13)

24. Antoni $\mathrm{J}$ et al OROS methylphenidate for treatment of adults with AHD Expert Rev. Neurother 2009,9(8):1121-1131

25. Dopheide JA et al Attention-Deficit-Hyperactivity Disorder: An Update Pharmacotherapy 2009;29(6):656-79

26. Trott G.A.: „Welche Folgen hat eine ausgebliebene angemessene Behandlung einer Aufmerksamkeitsdefizit-/Hyperaktivitätsstörung ?"Vortrag an der Tagung der sfg-adhs vom 8.9.07 auf dem Gurten in Bern

27. Sobansky E und Alm B, ADHS bei Erwachsenen. Ein Überblick Nervenarzt 2004, 75;697-716

28. Barkley R. A. (2009) Attention Deficit Hyperactivity Disorder in Adults. The latest assessment and treatment strategies Boston Jones and Bartlett Publishers

29. Dr. Miguel Marset, Genève (anciennement engagé au Centre des addictions des Hôpitaux Universitaires de Genève) „Le TDAH et les conduites addictives: diagnostic et prise en charge » Seminaire Lilly « Le TDAH se développe avec le patient », 25. Fév. 2010, Hôtel du Parc, Martigny

30. PD M. Bader, Lausanne, persönliche Mitteilung

31. Christophe C. Kaufmann, aufgrund fünfjähriger klinischer Erfahrung mit 125vorwiegend erwachsenen ADHS-Patienten

32. IV Gutachten von Frau A.S. durch Dr S, Lausanne,

33. Aufenthalt von Frau C. B. im HFR Freiburg 